\title{
Designing virtual natural environments for older adults in residential care facilities
}

\author{
Rikard Lundstedt $^{\mathrm{a}, *}$, Carita Håkansson ${ }^{\mathrm{b}}$, Mare Lõhmus ${ }^{\mathrm{b}, \mathrm{c}, \mathrm{d}}$ and Mattias Wallergård ${ }^{\mathrm{a}}$ \\ ${ }^{a}$ Department of Design Sciences, Division of Ergonomics and Aerosol Technology, Faculty of Engineering, Lund \\ University, Lund, Sweden \\ ${ }^{\mathrm{b}}$ Department of Laboratory Medicine, Division of Occupational and Environmental Medicine, Faculty of Medicine, \\ Lund University, Lund, Sweden \\ ${ }^{\mathrm{c}}$ Institute of Environmental Medicine, Karolinska Institute, Stockholm, Sweden \\ ${ }^{\mathrm{d}}$ Centre for Occupational and Environmental Medicine, Stockholm County Council, Stockholm, Sweden
}

Received 6 April 2021

Accepted 29 July 2021

\begin{abstract}
.
BACKGROUND: Spending time in natural environments is beneficial for mental health and cognitive function. Unfortunately, many elderly citizens, who would potentially gain from these beneficial effects, have limited or no access to natural environments. Virtual reality may provide the experience of being present in a natural environment without the need of transportation.

OBJECTIVE: The purpose of the present study was to acquire an understanding of how residents and staff at a residential care facility may use and experience different virtual natural environments, and thus aid the design of virtual natural environments.

METHODS: We used qualitative method, collecting data through interviews and observations. The data was analyzed using qualitative content analysis.

RESULTS: Exposure to virtual natural environments provoked various emotional reactions, and promoted skill development and social interaction among participants. The staff perceived virtual natural environments as potentially useful for, for example, improving residents' anxiety. We identified several user interface improvements.

CONCLUSIONS: The present study discusses how various aspects of virtual natural environments work in relation to the experience of a user and how this might affect wellbeing in older adults. These aspects may be valuable to consider in the design of virtual natural environments for use at residential care facilities.
\end{abstract}

Keywords: Elderly care, nature, virtual reality, well-being, qualitative method

\section{Introduction}

The proportion of elderly citizens in Europe is increasing. According to predictions from Eurostat, the ratio of citizens aged 65 and older compared with 15 - to 64-year-olds is expected to almost double by 2100 [1]. Since old age is generally associated with increasing

\footnotetext{
*Corresponding author: Rikard Lundstedt, Department of Design Sciences, Division of Ergonomics and Aerosol Technology, Faculty of Engineering, Lund University, 22100 Lund, Sweden. Tel.: +46 721640573; E-mail: rikard.lundstedt@design.lth.se.
}

health problems, this societal trend calls for interventions and adaptations focused on increasing the quality of life and wellbeing of the elderly population. Currently, an average European older than 65 years is expected to live, in average, eight of their 18 remaining years as unhealthy, entailing functional limitations due to physical, mental, and cognitive health problems $[2,3]$. The most common way to tackle the growing health burden associated with advanced age, has been to increase the use of different kinds of pharmaceuticals of an elderly individual. However, especially in case of mental and cognitive health problems, the effect of medications is often limited and may entail various nega- 
tive side effects [4]. Consequently, problems associated with polypharmacy, inappropriate medication use, and adverse drug reactions are common in the residential care of the elderly and may lead to, both, decreased life quality of the individual and increased health-economic costs for the society [5,6]. Encouragingly, deprescribing drugs and adopting non-pharmacological approaches to improve wellbeing in older adults has become an increasingly popular approach [7].

Today, abundant scientific evidence support the idea that exposure to natural environments such as forests, parks and beaches has a beneficial effect on cognitive function, mental health and wellbeing [8-13]. Unfortunately, many elderly citizens, who would potentially gain from these beneficial effects, often have limited or no access to natural environments due to limitations caused by different kinds of aging-related health problems.

Virtual Reality (VR) technology provides the means for an individual to get the experience of being present in different environments, e.g. forests, parks, beaches or other natural environments, without a need of transportation. VR has been suggested as a means for making nature experiences more accessible for older adults who have limited or no access to spending time in natural environments $[14,15]$. In the present study we investigated the workings of Virtual Natural Environments (VNE) at a residential care facility by inviting residents (and staff) to use VNEs under our observation.

\subsection{Concepts and features in VR technology}

Today, virtual environments (VE) are commonly created with the help of real time 3D graphics, i.e., computer game technology, or with 360-degree video footage that has been captured with an omnidirectional camera. Presence is a central concept in the VR theory, and describes a viewer's perceptual illusion of "being there" [16]. Viewing VEs through immersive technology such a head mounted display (HMD) provides a higher sense of presence compared to viewing VEs by using non-immersive technology such as a single TV or computer screen [16-18]. Another key element helping to create the feeling of presence is the sense of agency, which can be defined as the viewer's sense that they are the one causing an action [19] by, e.g., actively deciding what to look at and do, rather than passively watching a predetermined set of events. Thus, interactive VR has the potential to provide a higher sense of presence than non-interactive VR (for in-depth discussion about immersive VR see [20]).

\subsection{Previous use of VR in therapeutics}

Previous studies have suggested that use of VR may, in certain conditions, be an effective and safe alternative method of treating and preventing both mental and physiological health problems [15]. Interventions including use of VR technology in medical care has for example, been tested in rehabilitation of neurological disorders and stroke [21], in cognitive rehabilitation, addressing gait and cognitive deficits after brain injury [22,23]; and in treatment of psychiatric disorders such as post-traumatic stress disorder, anxiety disorders, phobias and pain management [23,24]. Research also cautiously encourage the use of VR technology in treatment of certain health problems frequenting among the elderly population. For example, a systematic review concluded that VR games can have positive effects on balance and fear of falling in community-dwelling older adults [25]. Another study indicated an increased positive attitude towards the use of immersive (HMDbased) VR technology, without any associated cybersickness by older adults after first use [26].

Thus, according to previous findings, it is possible that exposure to natural environments through immersive VR is a feasible way to overcome difficulties with accessing in-vivo natural environments among the elderly. The use of VNEs has been suggested as a means to increase mental well-being in older adults who experience reduced mobility $[14,15]$.

However, before the use of VNEs can be considered by residents of a care facility, they first need to acquire a certain level of knowledge about what VR is. Since VR essentially is a medium that speaks to the senses, it must be sensed to be understood. And just as any other experience, VR is perceived differently among different individuals [27]. Therefore, the experience of VR, cannot be fully conveyed through words or other media and must be obtained first-hand to be understood. Currently, more immersive HMDs such as the HTC Vive, compared to less immersive ones such as the Oculus Go, may appear bulky, uncomfortable and intrusive and may therefore deter from future use in individuals with cautious disposition. Furthermore, using highly immersive VR may be perceived as quite intense, potentially overwhelming at first experience. Thus, it is possible that frail individuals that tire easily, may find such experiences off-putting from future use. Using a less immersive HMD at first introduction of VR for older adults at residential care facilities, may therefore be preferable. 


\subsection{Use of VR natural environments in the elderly population}

There are several previous examples of studies that have involved viewings of natural environments by VR technology for various therapeutic purposes including: improving muscle strength and balance [28]; improving cognitive function [28,29]; reducing anxiety and agitation [30], and improving engagement, apathy, and mood states [31] in people with dementia; and improving motivation to exercise and mental wellbeing [32]. A recent study explored the acceptability of VNEs for people with memory loss at memory cafes [33]. Some of these studies have involved activities like kayaking [28], bicycling [29,32], or interacting with objects present in the VE through hand and arm movements [31]. However, a rather small number of the previous studies have regarded the potential therapeutic effects of exposure to natural environments. Furthermore, in the majority of the above mentioned studies the VR technology used is non-HMD and single screen-based [28,30,31], and/or non-interactive [28,30].

An exception from this trend is, however, the study published by Bruun-Pedersen et al. [32], which investigated the potential of increasing motivation to exercise in residents of elderly care facilities, by exposing them to virtual bike rides in VNEs. Bruun-Pedersen et al. based the design of their VNEs on a combination of knowledge derived from previous non-VR-related sources reviewing restorative aspects of natural environments and experiences from tourism and recreational design, as well as from VR-specific sources including examples from the areas of urban design and urban planning in VR, and spatial recognition for navigation in VNEs.

In the present study we used a different approach to Bruun-Pedersen et al. by involving the end-users in the process to design the VNE. We based our approach on the following arguments. VR provides a perceptual but not cognitive illusion of being in an different reality [16], i.e., it feels real, but the users are aware that it is an illusion. Therefore, when a user perceives an artifact in VR, the VR medium itself is likely to be on the user's mind; for example, the artifact may be viewed in light of the VR system's capability to produce an illusion of it. Thus, the VR medium is an essential part of the context in which the artifact is perceived. Since a user's perceived meaning of an artifact is dependent on the context in which it resides [27], and VR by definition is fundamentally different from actual reality, a user's perceived meaning of natural environments in a VR context is likely to be essentially different to the same user's perceived meaning of real natural environments. Therefore, knowledge of restorative natural environments such as the works by Kaplan [34] or Bengtsson and Grahn [35] may not be valid for, and/or transferrable to, VNEs. Furthermore, inspired by Krippendorff and Butter [27], we do not believe one can design VNEs with inherent meanings. Users find their own meanings in artifacts through using them and communicating about them with others. Thus, a designer of VNEs can only, based on their understanding of the users' perceptions, attempt to provide the means for users to find meaning in VNEs. Therefore, in order to design VNEs that are meaningful for users, we believe it is necessary to invite the participation of users into the design process.

\subsection{VR Island}

In accordance with above presented reasoning, authors of the present study have previously carried out the first iteration of a design process in which elderly users participated (Lundstedt R, Persson J, Håkansson C, Frennert S, Wallergård M, unpublished data). By observing the participants' actions while using a VNE prototype, and by listening to their articulated thoughts, suggestions and preferences, we acquired an understanding of their perceived meanings of the prototype, which informed our design decisions as the development proceeded. This process led to the creation of a VNE, which was named "VR Island". VR Island is designed in a way that gives the viewer ability to freely explore, and to some extent, manipulate the environment; or, if they so choose, idly observe the scenery. Further, by including a separate TV-screen showing a third-person view of the HMD-wearing viewer's avatar in the VNE, VR Island allows a group of by-standing residents to be somewhat co-present in the VNE and thus engage in social interaction with each other and the current viewer. According to a recent publication reviewing indoor nature interventions in the residential care setting [36], the interventions that entailed some active involvement were more likely to exert beneficial health effects than passive exposure only. Further, the authors of the review recommended that for added benefits, future indoor nature interventions should provide social stimuli such as shared/group experiences, skill development and decision making. VR Island is described further in Section 2.3.3.

Our previous work with the development of VR Island (Lundstedt R, Persson J, Håkansson C, Frennert 
$\mathrm{S}$, Wallergård $\mathrm{M}$, unpublished data) was carried out in a laboratory environment and only included elderly study participants in good condition who were able to, without assistance, transport themselves to the lab. Thus, immobile elderly individuals in frail condition were excluded from the study, which is unfortunate as this group is the one that is most likely to experience a shortage in exposure to natural environments and may thus benefit the most from a VR intervention. Also, as stated above, a user's perceived meaning of an artifact is dependent on the context [27], e.g., laboratory environment versus residential care facility. These conditions were deemed acceptable, when the trade-off between authenticity and operability were considered, for the purpose of producing a prototype to serve as a starting point for coming iterations. Furthermore, the resident wearing the HMD is not the only stakeholder. An artifact such as a VNE at a residential care facility is the concern of a network of different stakeholders, e.g., residents, assistants, activity workers, nurses, next of kin of residents, caretakers, administrators, purchasers, sanitary technicians and property managers. Stakeholders form and coordinate, in communication with each other, their meanings of an artifact based on their individual perspectives [27]. Therefore, the purpose of the present study was to acquire an understanding of how residents and staff at a residential care facility may use and experience different virtual natural environments, and thus aid the design of virtual natural environments.

\section{Methods}

For this study, we used qualitative method, collecting data through interviews and observations. The data was analyzed using qualitative content analysis.

\subsection{Participants}

We contacted an administration for elderly care facilities in a southern Swedish municipality. This particular municipality was chosen because of the relatively diverse socioeconomic background among its population. The administration expressed interest to participate in the study and conveyed contact with one of their residential care facilities with a suitable residential profile.

Our initial plan was to create two groups of participants, each consisting of four to six residents (group A and B). According to our exclusion criteria a person that was bedridden, suffered from severe dementia, or prone to motion sickness, was not suitable to partake. In addition, we only accepted participants that could speak and understand Swedish, had sufficient eyesight to watch television, sufficient cognitive ability to perceive the VNE and to answer questions about their experience, and were generally fit enough to be able to handle and potentially benefit from the VR experiences. In the selection of study participants, we relied on the help from the care home staff, since they were familiar with the inhabitants, their abilities and health status. The residents who were suitable to partake in the study first received brief information about the study including general information about what VR is, what participating in the study entails in practice, and potential risks of participating. Individuals who stated their interest to participate then received further information including the purpose of the study and the participants' rights regarding confidentiality and voluntarism. Persons who remained interested in partaking were thereafter asked to sign their written informed consent.

\subsection{Study procedure}

In order to create a familiar and comfortable situation for the residents, we based our VR test sessions on a traditional social activity in Sweden - having coffee. In practice, this meant that we sat down, drank coffee and ate pastries together with the participants as we invited them to experience VNEs through the VR devices we had brought. At least one member of the staff partook in the sessions, assisting the residents when necessary. Each VR coffee session lasted approximately one hour during which each participant spent between 5 and 20 minutes immersed in VR, wearing an HMD.

During a two-week period in the spring of 2019, Group A participated in two VR coffee sessions per week resulting in four sessions in total (see Fig. 1). In order to achieve a, for the participants, manageable progression towards advanced VR use, we introduced VNEs in ascending order in terms of levels of immersion and interactivity (see Section 2.3). After our final session with group A, we paused the study for three weeks before performing the same sequence of sessions with group B. The reason for the break was to allow us to, based on what we had learned from the VR coffee sessions with group A, make changes to our test procedure and VNE design before the VR coffee sessions with group B began.

\subsection{Experimental setup}

In addition to VR Island, we included two other 


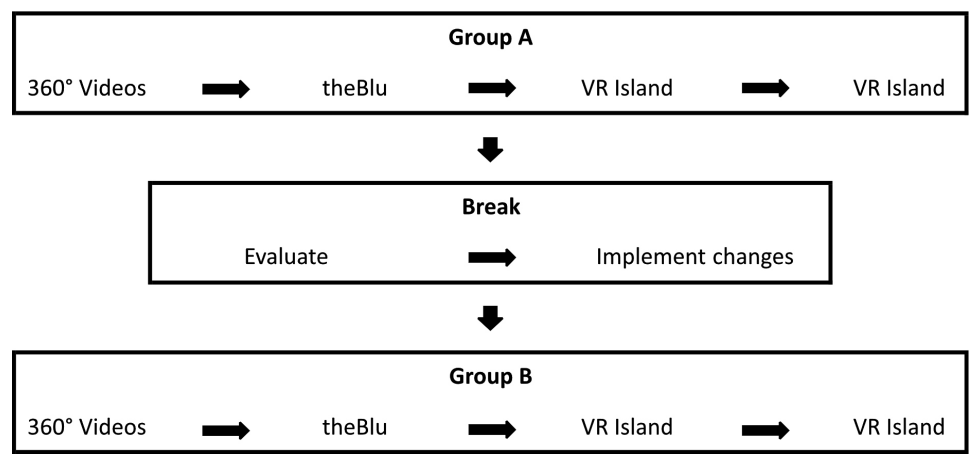

Fig. 1. Study procedure.

VNEs to our study (see detailed descriptions below). This would later allow us to combine traits of the different VNEs that appeared to be important for a positive viewer experience in future versions of VR Island. The participants used three types of headsets each paired with their own specific audiovisual VR content. These specific content-device combinations were: a) $360^{\circ}$ blue space videos $-360^{\circ}$ videos of coastal environments [33], viewed on Oculus Go headsets; b) the$B l u$ - a series of interactive under water environments rendered using real time 3D graphics (Wevr, USA), viewed on a Samsung Odyssey headset; and c) VR Island - an interactive explorable island featuring various types of natural environments rendered using real time 3D graphics, viewed on an a HTC Vive headset. With all three HMDs, a pair of brand-specific cableless handheld controllers were included for user interaction.

\subsection{1. $360^{\circ}$ blue space videos on Oculus Go}

Oculus Go is a lightweight cableless standalone display that can be strapped onto the viewer's head, alternatively held in the viewer's hands and looked into as in using a pair of binoculars. The $360^{\circ}$ blue space videos are filmed at various coastal locations in Cornwall, England [33]. Experiencing these videos through an Oculus Go headset offers the viewer three degrees of freedom, which means that the headset tracks the viewer's head orientation and the viewer is able to view the scenery from any direction by turning their head. However, if the viewer shifts the position of their head, for example by moving it sideways, that movement will not be reflected in the display.

\subsection{2. theBlu on Samsung Odyssey}

Samsung Odyssey is a head mounted display that requires a cable-connection to a computer. theBlu is a series of interactive underwater environments rendered in real time. While viewing, the viewer will find themselves in an underwater environment such as a shipwreck or a reef. The environments feature scripted events such as marine animals that may swim by or approach the viewer. For example: sea anemones, sea turtles, various species of fish, a swarm of jellyfish and an 80-foot whale. The viewer is able to interact with animals by poking them, whereon they may get spooked and flee.

theBlu offers six degrees of freedom which means that not only can the viewer turn their head to look in any direction but they can also shift their location, for example, look behind a rock, bend down in order to closer inspect an underwater plant, or duck to avoid an intrusive fish. However, the user cannot move beyond the area of the current scene, which covers only a few meters.

\subsubsection{VR Island}

Like Samsung Odyssey, the HTC Vive is an HMD that requires a cable-connection to a computer. VR Island is a virtual island with various types of environments for a viewer to explore, such as a beach with photorealistic water wave simulation, a meadow and a small rowboat in calm water (see Fig. 2). The island also contains birds, fish, butterflies and a lighthouse. Also, in one spot on the Island there is an apple tree from which viewers can pick apples and put these in a basket placed underneath the tree.

Similarly to theBlu, VR Island offers the viewer six degrees of freedom, but in addition a possibility to freely move around and explore the entire Island. Designed to accommodate for cognitive, physical and motor function impairments associated with old age, locomotion in VR Island is done seated through a form of torso-directed steering with a minimal number of unintegrated (unnatural) control dimensions [37] (see Fig. 3). The viewer typically sits in a swivel chair which is tracked by the VR-system that constantly monitors 


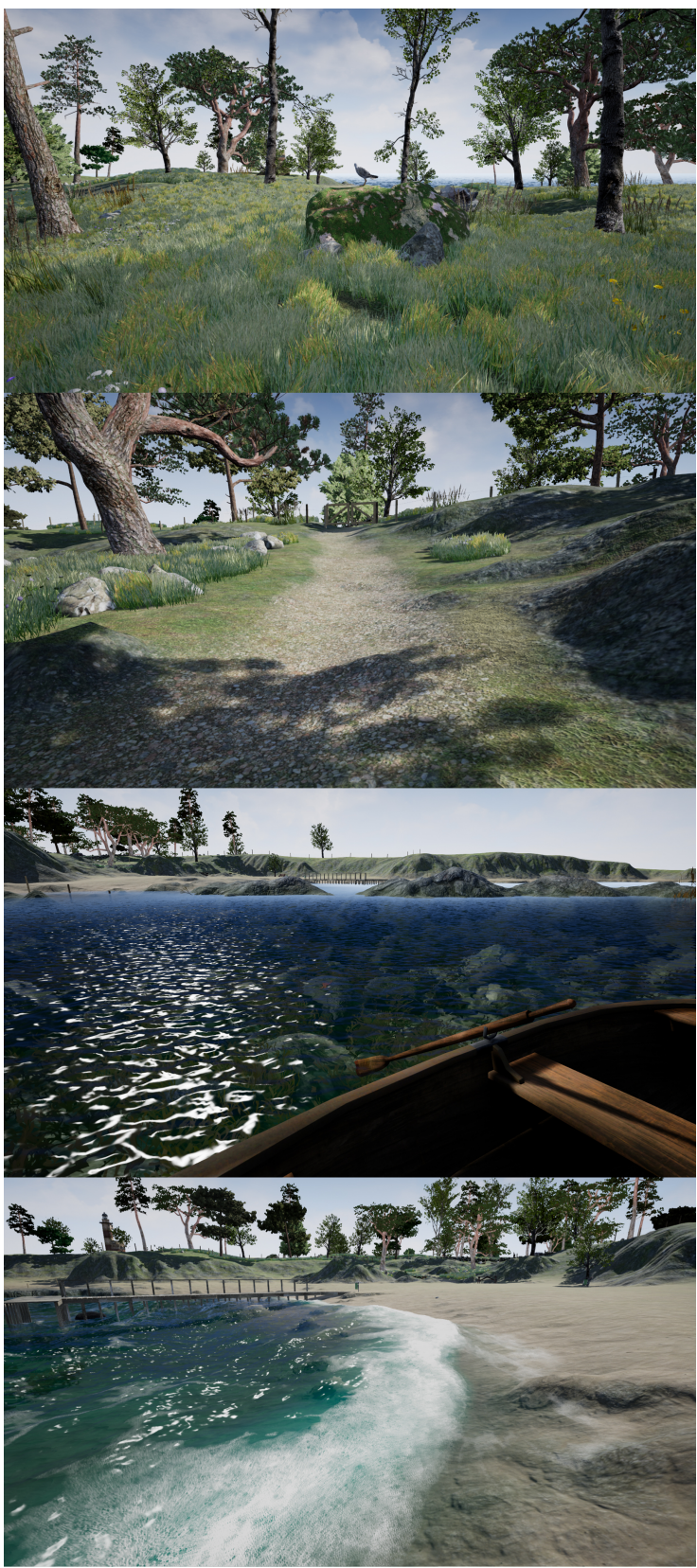

Fig. 2. VR Island environments.

the chair's position and orientation. This was made possible by attaching of one of the handheld controllers to the chair. As a result, the chair can be used as a sort of vehicle in the VNE. The viewer can steer by simply turning in the direction of where they wish to go and press a button on the handheld controller which acts as an accelerator. To avoid cyber sickness, acceleration, deceleration and forward propulsion speed is, by the design, slow [38].

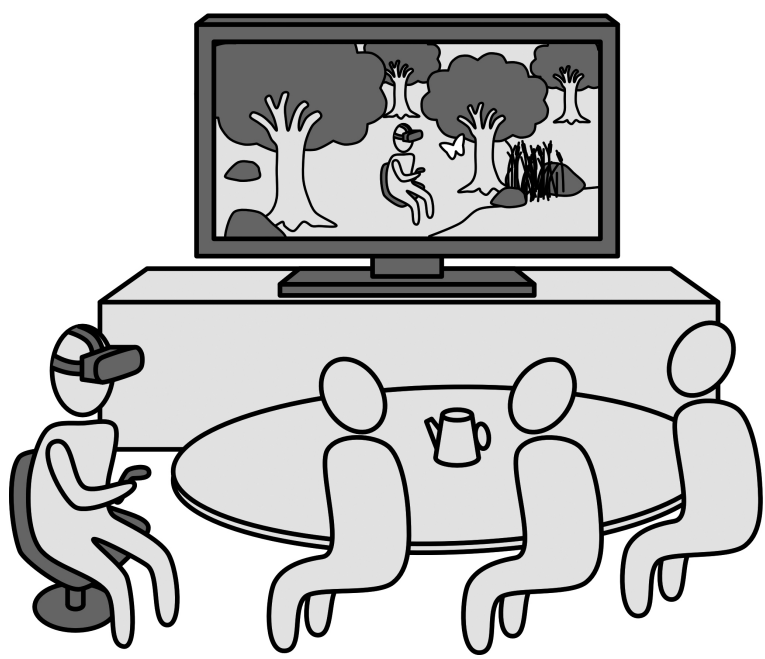

Fig. 3. VR Island setup.

By placing a large monitor (LED TV) display centrally in the room, a "spectator view", corresponding to the view of the HMD-wearing viewer, is provided for the by-standers (see Fig. 3) allowing these to follow the viewer's avatar on the nature trek. An analogy envisioned by the authors during development was that the bystanders should be as the passengers of a safari truck, driven by the HMD-wearing resident.

\subsection{Data collection}

During the VR coffee sessions, we took notes of observations of the residents' reactions as expressed through oral communication, and verbal and bodily expressions. We also noted our observations of the usability of the VNEs and the situation in general. After each VR coffee session, each participating resident was, separately from the group, asked a series of open-ended questions by a staff member about their experience. After the final VR session, we interviewed two of the staff members who had been the most frequently involved in the VR sessions regarding their views of the usefulness of such VR sessions at residential care facilities and their general impressions. The interview was audio-recorded and later transcribed. The staff were also asked to respond to questionnaires regarding the participating residents' gender, age, number of years residing at the facility, and diagnose/reason for taking residence at the facility.

\subsection{Analysis method}

To allow for methodological triangulation [39], the unit of analysis comprised of our notes of observations, 
the answers to the open-ended questions by the participants, and the interview with the staff. The first author (RL) transcribed the audio recording of the interview with the staff. All documents were shared with all members of the research team. We analyzed the data through an inductive qualitative method inspired by Graneheim and Lundman [40].

We read through the above mentioned documents several times to get an understanding of the content as a whole. Using NVivo, the first author broke down the text into meaning bearing units. The units were grouped, whereon categories and subcategories (types of reactions, impressions, ideas, opinions and other expressions) emerged through reflection on their meaning. During the analysis, the authors made comparisons with the original texts and discussed the analysis. In an iterative manner, the team went back and forth several times between the first author revising the analysis in NVivo and the whole team meeting and reflecting in group until the team had reached an agreement on the analysis.

\section{Results}

Nine residents registered to participate in the study. However, two of them did, for unknown reasons, only attend one VR coffee session. Thus, the final sample resulted in seven residents consisting of four women and three men ranging from 67 to 91 in age with a median age of 88 . The number of years the participating residents had lived at the residential care facility ranged from 1 to 6 with a mean value of 3.4. The study participants were diagnosed with various mental and/or physical health conditions, which, in most cases, were the initial reason behind moving to a care facility.

Although we provided twice as many VR Island sessions as $360^{\circ}$ video and theBlu sessions, varying turnout by the participants resulted in a fairly equal number of uses of the three different VNEs. As a result of analyzing the observations and the interviews, six categories emerged: emotional reactions, expressions of interest, progression, social interaction, perceived usefulness and usability matters. The $360^{\circ}$ videos worked well as an entry level VNE. All study participants who tried the $360^{\circ}$ videos were able to use the Oculus Go to become immersed in the content and acquire a basic understanding of VR. Further, we observed no signs of any participant becoming overwhelmed or fatigued by the $360^{\circ}$ videos or any subsequent, more immersive VNEs.

\subsection{Emotional reactions}

The participating residents expressed emotions during the VR coffee sessions: a) spontaneously, while immersed in a VNE wearing the HMD; b) in conversion with other study participants, staff and researchers while standing by, observing others exploring VNEs; and c) in interviews after sessions. In addition, some expressions of emotional reactions by participating residents were noted by staff in between sessions, and were reported to us regularly. The number of observed emotional reactions was the highest for theBlu, slightly lower for VR Island and noticeably lower for the $360^{\circ}$ videos.

\subsubsection{Aesthetical pleasure}

Participating residents expressed, through oral communication, their enjoyment with the aesthetical qualities of the VNEs. Most expressions reflected a general impression of what the participant looked at in the moment, not specifying any particular aspect of the VNE, e.g., "It's so pretty". Only a few expressions addressed specific objects or aspects of the environment like the colors, flowers, sea, grass or sky. Most expressions of aesthetical pleasure were uttered in relation to VR Island. Comments about a boat or trees being ugly also occurred.

\subsubsection{Joy}

Study participants expressed joy both non-verbally through giggling, laughing and smiling, and through verbalizations, e.g., "that was fun". These expressions were noted both during and after the participants had been immersed in the VNE during the VR coffee sessions. Most expressions of joy were registered in association to theBlu.

\subsubsection{Fascination/mind-blowing realization}

As we interpreted their verbalizations and body language, participating residents expressed a fascination, sometimes raised to the level of "mind-blowing", for the capabilities of VR technology, the experience of the natural environment and its content, and/or the strong feeling of presence. E.g., "It's amazing what you can come up with”, “Totally amazing, totally unbelievable!”, “Oh my, that's a long sucker!" (while viewing the whale in theBlue). Expressions of fascination were most commonly shown during or in direct connection with being present in theBlu.

\subsubsection{Discomfort}

Expressed through verbalizations and sometimes 
through non-verbal expressions such as flinching, residents appeared startled or to experience mild discomfort when accidentally driving through or too close to objects like trees or fences in VR Island (collision detection for such objects had been disabled for reasons described in Section 3.7.1). In addition, we registered occasional signs of discomfort in study participants expressing that the water in VR Island appeared cold and stormy. However, these experiences were not necessarily negative, but often pleasurably thrilling and exciting. This became especially evident in theBlu when residents expressed these feelings while coming in close contact with marine animals such as giant jellyfish and the whale.

\subsubsection{General positivity}

Other less distinctive positive responses signifying appreciation for, contentment with, or an otherwise positive attitude towards the VNEs were expressed by the participants. We classified these responses, expressed as comments on the general experience or some specific content or aspect of the VNE being "nice", "not bad", "cool" or "wonderful", as "general positivity". Most generally positive reactions were expressed in relation to theBlu.

\subsubsection{Negative reactions}

In addition to the above, we observed negative reactions: anger, based on a wish to go outside and breathe fresh air instead of using VR; boredom in association with using the $360^{\circ}$ videos; and dissatisfaction with the slowness of the propulsion speed while exploring VR Island.

\subsection{Expressions of interest}

During the VR coffee sessions, residents showed both positive and negative interest in VNEs. The number of observations of positive expressions of interest outweighed the negative ones. Positive expressions of interest include comments by participants where it is unclear whether these were actually connected to using the VNEs or if they were uttered in regard to something else, e.g., interest to participate in the VR coffee sessions for the sake of the social interaction, or general curiosity about VR technology. However, the majority of positive expressions of interest were clearly associated to the interest of using the VNEs, or even interest in viewing specific content in the VNEs.

\subsection{Usability matters}

During the VR coffee sessions and the interview with the staff, we made several observations of usability matters. Some regarded the comfort of the HMDs where the Oculus Go HMD was considered flexible and smooth, being cable-less and lightweight. Also, there were observations of the HTC Vive HMD being experienced as heavy, slightly painful on the face and/or unstable, sliding down.

\subsubsection{Problems associated with the use of handheld controllers}

Some participants had difficulties using the handheld controller of VR Island. The difficulty laid in using the index finger button on the controller that works as an accelerator. It is possible that, since the viewer could not see their own hands on the controller, it became difficult to locate the button. In addition, there are several other buttons on the controller which may have distracted finding the accelerator button. It is also possible that some participants suffered from impaired fine motor function. However, some residents did not appear to have any difficulties with using the controller.

\subsubsection{Difficulties with turning in VR Island}

Some participants had difficulties steering by turning the swivel chair using their feet. Further, some residents in the second group who were wheelchair users were not able to use the swivel chair at all. As we realized this early in the study, we were able to implement a solution during the mid-study break (see Section 3.7.2). However, some participants appeared to have no problem with steering using the swivel chair.

\subsubsection{Staff can steer for residents in VR Island}

In cases when the study participants could not steer themselves, a staff member was able to assist, turning the chair and managing the handheld controller. Since the staff member seemed to be able to efficiently communicate with the participants and interpret their wishes, it gave the resident a degree of agency. Researchers, who were not as familiar with the residents as the members of staff, had a harder time communicating and conveying agency when assisting with steering. It appeared useful to view the spectator view as well as the VR computer's monitor (displaying the VNE from the perspective of the participant) as this gave the assistant a view of what the resident saw and at the same time the surrounding environment. However, there were occasional problems, associated with the assistant blocking the controller mounted on the chair with their body, so that the system temporarily lost tracking. Furthermore, according to the staff, turning the wheelchair with 
one of their hands occupied by a controller, was rather cumbersome and heavy.

\subsubsection{Inability to see what the residents see in Oculus Go}

Based on our own observations, but also on the information emerging from the staff interview, a factor that made assisting a resident wearing the Oculus Go HMD difficult, was that the assisting person (staff) was not able to see what the person saw. In order to prepare the Oculus Go for viewing by a resident, an assistant is required to wear the HMD to inspect the graphical user interface while performing various maneuvers using a handheld controller. Once the assistant has taken off the HMD and the resident has put it on, mishaps or technical errors may occur that is beyond the assistant's control. E.g.: the device has crashed; the content is shown in poor resolution or in an otherwise unfavorable way; or the content is replaced with something not intended. The assistant is thus fully relying on the reports of the viewer and can only help when the viewer reports an error.

\subsection{Perceived usefulness}

Based on the staff's observations of positive development cases among the participants during the study, including reduced need for on-demand medication, they projected that VNEs could be useful for reducing anxiety. Further, the staff reasoned that VNEs may reduce the use of on-demand medication, and thus both increase health in residents and reduce costs of residential care. In addition, they speculated that VR could help patients with neurological conditions, sorrow, fear of death and/or worry for survivors to temporarily escape from their troubles through occupying their thoughts with something else. Other uses of VNEs projected by the staff was as a complementary alternative for residents who cannot go outside as much as they would like, and that VR may incite residents to engage in physical activity, e.g., through virtual apple picking.

\subsection{Progression}

Some participants appeared to increasingly enjoy the VNEs as a result of repeated attendance of VR coffee sessions. Also, as the study proceeded, individual residents progressed in various ways relating to their VNE use. For example, we observed how residents' reactions to things they had previously been startled by, e.g., close encounters with marine animals, became calmer. Further, some residents became more skilled in steering and using the handheld controller while in VR Island.

\subsection{Social interaction}

In the beginning of the study, though most participants expressed themselves and commented what they saw while being in the VNEs, there was very little social interaction between the residents. However, in the later sessions social interactions became increasingly more common and all study participants in the room watched the spectator view screen attentively and commented and discussed what they saw. Both the by-standing residents (BSR) and the HMD wearing resident (HWR) as well as the assistant (A) were involved in conversation.

Example:

HWR: It's pretty with flowers.

BSR: Are those lupines?

HWR: I don't know what they're called but they're pretty.

A: Do you want to sit in a boat in calm water?

BSR: Calm??? It was rocking considerably!

Further, when asked the question "How did it feel to be in the group?" in the post-session interviews, answers described positive feelings, e.g., fun, good, comfortable, nice and cozy. There were also more neutral answers such as "Nothing special" and "Okay".

\subsection{Mid study changes}

We adjusted the VR Island prototype, according to the feedback while the study was ongoing. The following changes were made:

\subsubsection{Collision detection}

As described in Section 3.1.4, we observed apparent discomfort and flinching in study participants during their unintentional driving straight through objects such as trees, fences and rocks in the VNE. In our original design, collision detection for such objects was disabled for the purpose of avoiding problems with users getting stuck and having to go around objects, or being obstructed, for example, when driving to close to a tree. However, after making these new observations, we decided to turn on the collision detection functionality of this type of objects, making it impossible to drive through them. In preceding sessions, we did not make such observations again. 


\subsubsection{Wheelchair support}

To make it possible for wheelchair users to explore the VR Island environment we implemented a function that allowed an assistant to attach the chairtracking controller to the wheelchair and recalibrate it by a couple of keystrokes on the computer. As a result, wheelchair users were able to explore VR Island (through assistance) without getting out of their wheelchairs.

\section{Discussion}

According to our observations, introducing VR technology and VNEs as a potential diversification to everyday routines in the elderly care settings is a feasible intervention, which may help to mitigate boredom and increase the quality of life in residents. While using the VNEs, participating residents appeared to experience much joy and fascination. Often, they found the environments aesthetically pleasing. They were thrilled by encounters with scary but gentle sea creatures and appeared to enjoy mind-blowing experiences. Generally, the participants showed interest in using the VNEs and expressed a high appreciation for them, with a few exceptions.

Over the course of the study, we observed increased confidence and VR interaction skills in participants. Further, the spectator view, which allowed bystanders to join in on the VNE experience, encouraged social interaction between the participants. The staff perceived VNEs as potentially useful for improving residents' anxiety, reducing on-demand medication, motivating exercise and offering complementary alternative outdoors experiences.

All residents participating in our study were able to explore VR Island, either independently, or through assistance. During the study, we identified possible improvements to the user interface, both from the resident's and the assistant's perspective.

\subsection{Interest}

Our observations of the participants' expressions of interest suggest that there was a genuine interest to use VNEs among the participants - that it was not merely something else, surrounding the use of VNEs that drove their interest. Negative reactions and expressions of disinterest, which also occurred, may suggest that VNEs does not suit everyone. Possible explanations may be a lack in modalities - failure to provide sensations such as air movement, temperature and smell, i.e., fresh air; and a lack in diversity - failure to meet individual residents' preferences in content. It is possible that due to such shortcomings, the VNEs failed to provide the means for individual participants to acquire a meaningful experience. However, based on participants' apparent increased interest, skills and enjoyment after acquiring extended experience of using VNEs, we hypothesize that it may take longer than we had originally anticipated to get used to VR and that VNEs may be more beneficial for individual residents after getting used to the experience of VR.

\subsection{Usability matters}

In order to counter the difficulties participants had using the handheld controller while exploring VR Island (see Section 3.3.1), an alternative controller could be designed with a single easy-to-push button. Thus, the user would no longer risk choosing the incorrect button. In addition, the controller could be designed to accommodate for impaired fine motor function, e.g., for a grip action. Regarding the difficulties the members of the staff experienced while turning a wheelchair with one hand occupied by a controller (see Section 3.3.3), possible solutions may include to design an easy-torevolve platform that a wheelchair can be easily rolled up onto, and/or to redesign the controller so it can be fitted onto a wheelchair handle.

As previously stated, in the case of a resident using an Oculus Go, an assistant is fully relying on the viewer's reports of any error since the assistant cannot see what the resident sees (see Section 3.3.4). Concerningly, first-time users, who do not know what to expect, may accept the compromised content and may not raise any complaints; or, they may be unable to communicate for other reasons. Not only may the then ongoing experience be negatively impacted, but the onboarding of the resident to future VR experiences may also be compromised, which may lead to missed opportunities for the resident.

On certain occasions when participants had been present in a VNE for a while and wanted to finish, they had to ask us how to exit VR. "How do I come back, to reality?" It made us realize that we had not devised a method for the residents to exit VR by their own power or initiative. Routinely, the HMD was mounted and removed by an assistant. Therefore, though the residents may be physically capable of removing the HMD, they may not be inclined to do so on their own accord. This may also be difficult to do by yourself. Further, viewers 
may be so immersed that they temporarily forget that they are wearing the HMD. Therefore, participating residents were generally left to call upon someone on the outside to help them exit VR. But we had not established the concept of exiting VR with the participants and therefore they may have lacked the tools to communicate their intent to do so. In essence, it is necessary to device some sort of easily accessible return path from VR, be it through established verbal communication protocol, or through the user interface, or both.

\subsection{Gradual progression}

Gradual progression appears to be instrumental for the residents' beneficial use of VNEs. Our introduction progressed slowly and stage-wise. On their first VR coffee session, participating residents were able to get a basic understanding of what VR entails through a first-hand experience, using a less immersive/intrusive HMD. It being their first encounter with VR, the choice of such an HMD may have helped the onboarding process. Furthermore, the benefits of using VNEs seem to increase as the residents become more experienced. The progression itself, in the form of learning, skill development and acquiring experience, is likely to be a beneficial factor in the VNE use [36] since lifelong learning has been suggested to promote psychological wellbeing in older adults $[41,42]$.

\subsubsection{Entry level VNEs}

Our observations suggest that VNEs with a lower level of immersion, lack of interactivity and tranquil sceneries, such as the $360^{\circ}$ videos, may be most suitably used at the initial level during the VR introduction process. The notably lower frequency of voiced emotional reactions by the study participants while using the $360^{\circ}$ videos, compared to when they were using other VNEs, suggests that the risk of becoming overwhelmed by the $360^{\circ}$ videos is low. However, there is a risk for the $360^{\circ}$ videos to be an underwhelming experience, misrepresent the true capabilities of VR, and thus affect onboarding negatively.

\subsection{Diversity in VNEs}

The expressions of fascination/mind-blowing realization and joy were more frequent when people were viewing theBlu than when using VR Island. This was not so surprising since we found theBlu to be somewhat reminiscent of theme park rides, which are designed to invoke such feelings. In theBlu, the viewer does not need to use their own initiative, but is instead served close encounters with various awe-inspiring marine species, which amounts to a rather spectacular show. The emotional responses of the study participants to theBlu were interpreted as highly positive.

During the VR Island sessions, we observed that while some residents were eager explorers, some preferred to remain in one place, passively observing the scenery with mostly nothing happening. Therefore, future VNEs should provide the content to accommodate both for active exploration (with possibilities of imaginative discovery) and passive observation, accommodating for various levels of intensity on a scale from "nothing happens" or "find interest in the little things" type of content to mind-blowing. Further, we argue that VNEs should accommodate for individual preferences for various types of content such as different types of natural environments.

\subsection{User-friendliness versus authenticity}

The slightly lower number of emotions expressed by the participants while exposed to the VR Island environment compared to theBlu can be explained with the lower intensity, and more contemplative, inward directed influence by VR Island. As VR Island is interactive, it requires a certain degree of concentration and involvement. The users of VR Island must seek out their own thrills, which are admittedly milder than what is provided by theBlu, but is also truer to an authentic nature experience typical for Northern Europe where nature is not "user friendly", but rather unpredictable and elusive, e.g., you cannot spot an elk on demand. While walking in real nature, encounters with wild mammals are rare and therefore valuable - something to write home about. Contrarily, theBlu brings immediate gratification through a maximized and compressed spectacular show. The viewer does not have to do anything and is being served awe-inspiring content at a constant rate. Thus, it is reasonable to expect that theBlu will generate more emotional reactions over a shorter time period.

\subsection{Active involvement and decision making}

Although some usability issues need to be resolved, it is evident from presented findings that elderly study participants were in general able to be actively involved and exercise decision making by deciding where to go and what to do in VR Island, either under their own steam or through assistance. In cases when the study partici- 
pant needed assistance with steering, it appeared to be easier for the staff members than for the researchers to help residents convey their agency. It is likely that the staff's knowledge of the residents, and the relationships of trust they may have developed [43], helps the staff's ability to interpret the resident's emotions and wishes. Further, residents may be more comfortable with giving instructions and making demands to a person they know than to a previously unknown individual.

\subsection{The spectator view}

Experiencing indoor nature in group and during shared activities may promote the wellbeing of elderly more than individual indoor nature activities [36]. The spectator view seemed to make it possible for the residents to be social with each other within the context of the VNE. Being co-present in the VNE may promote the perception of a shared experience even further, allowing residents to reminisce together later, about their adventures in the VNE. Comments uttered by the bystanding study participants, while watching their fellow resident's avatar in the virtual environment, indicate that the spectators were able to, in some sense, follow along on the experience of the immersed viewer. However, with the spectator's screen being non-immersive, the by-standing residents probably did not feel as if they were present in the VNE. Further, no visual representation of any other resident existed inside the VNE for the HMD-wearing resident to notice. Thus, even though there was some social interaction between them and the rest of the group, the HMD wearing resident was most likely getting the experience of being the only one present in the VNE.

\subsection{Possible pathways from VNE use to improved mental wellbeing}

During our study we saw indications of VNE use being able to improve the mood of care home residents, at least in the short term. Previous literature has suggested that VNEs may potentially have a beneficial effect on mental well-being in older adults $[14,15]$. Our observations are in agreement with the previous literature, and thus allow us to speculate about potential positive health effect of a similar but more long-term intervention. However, further research is needed to examine whether VNE use can lead to improved mental well-being.

As a resident first begins to use VNEs, they may get a sense of having discovered a whole new world inside the HMD. The experience may arouse curiosity and an interest to explore such new worlds and the possibilities they may bring. Driven by this curiosity, the individual may develop new skills and experiences through their continued use of VNEs. Acquiring new skills and experiences may instill a sense of accomplishment and pride; also, gaining new interests and goals may bring a sense of added meaning in life. As the resident is present in the VNEs, they may get both contemplative and mind-blowing experiences, which may instill a sense of wonder, potentially broadening their perspectives. Such experiences may allow a troubled person, perhaps stuck on obsessing on their problems, to see beyond themselves and their troubles; or at least, temporarily take a break from them. Further, the ability to freely explore the environment may provide a sense of regained freedom and control to a person who otherwise experience reduced mobility. Experiences in VNEs may become memories that may reside in the resident's thoughts, potentially enriching their inner life and shared in social interaction with others.

\subsection{Risks}

There is a risk that VNEs are used as a replacement for providing real outdoor excursions for residents, e.g., in order to save resources. Or, that residents experience them as such. Therefore, it is important that it is understood by all the stakeholders involved that VNEs do not replace spending time in nature, but should rather be regarded as a complement.

\subsection{Limitations of the study}

Due to the limited time of the study we cannot see how extended use affects the stakeholders' meanings of the VNEs. Further, it is possible that other factors than VNE use lie behind some of the effects we saw, e.g.: something different happening - a break from the usual activities; attention - researchers are interested in them; or the social interaction during the sessions, unrelated to the VNEs. Also, there is a risk that the selection process favored those with positive attitudes towards trying new technologies such as VR, and that it may have affected the results. Further, since we did not measure effects on the residents' well-being, the results serve mostly as guidance for future development and study where well-being may be measured.

\subsection{Future research}

In order to see how VNE use affect mental wellbeing 
and on-demand medication use in older adults at residential care facilities, longitudinal interventions where both physiological and psychological effects are measured is needed. Further, this study has exposed several new challenges/questions:

- How does one introduce VR in a way that enables residents to acquire an authentic understanding of what VR is without inducing a too intense experience and at the same time not understating the capabilities of VR?

- How would additional modalities like the sense of temperature, air movement and smell affect the experience of VNEs?

- How would the simultaneous use of multiple HMDs, allowing residents to meet and interact within a VNE, affect their sense of co-presence, and how would that affect social behavior among residents?

\section{Acknowledgments}

We would like to thank all the participants as well as the staff members and administrators involved in making this study possible. This study was funded by European Union's Horizon 2020 research and innovation programme under grant agreement No 666773.

\section{Author contributions}

CONCEPTION: Rikard Lundstedt, Carita Håkansson, Mare Lõhmus and Mattias Wallergård

PERFORMANCE OF WORK: Rikard Lundstedt, Carita Håkansson and Mattias Wallergård

INTERPRETATION OR ANALYSIS OF DATA: Rikard Lundstedt, Carita Håkansson and Mattias Wallergård PREPARATION OF THE MANUSCRIPT: Rikard Lundstedt and Mare Lõhmus

REVISION FOR IMPORTANT INTELLECTUAL CONTENT: Rikard Lundstedt, Carita Håkansson, Mare Lõhmus and Mattias Wallergård

SUPERVISION: Mattias Wallergård and Carita Håkansson

\section{Ethical considerations}

Ethical approval for this study was obtained from the Regional Ethical Review Board of Lund, Sweden (2018/864). Informed consent was obtained for all study participants.

\section{Conflict of interest}

The authors have no conflicts of interest to report.

\section{References}

[1] Eurostat. Projected old-age dependency ratio [online dataset]. Luxembourg City: European Commission [updated $2021 \mathrm{Feb}$ 8; cited 2021 Mar 30]. Available from: https//ec.europa.eu/euro stat/databrowser/view/tps00200/default/table.

[2] Eurostat. Healthy life years at age 65 by sex [online dataset]. Luxembourg City: European Commission [updated 2021 Mar 11; cited 2021 Mar 30]. Available from: https//ec.europa.eu/ eurostat/databrowser/view/tepsr_sp320/default/table.

[3] Eurostat. Life expectancy at age 65 by sex [online database]. Luxembourg City: European Commission [updated 2021 Mar 30; cited 2021 Mar 30]. Available from: https//ec.europa.eu/ eurostat/databrowser/view/tepsr_sp330/default/table.

[4] Davies EA, O'Mahony MS. Adverse drug reactions in special populations - the elderly. Br J Clin Pharmacol. 2015 May; 80: 796-807. doi: 10.1111/bcp.12596.

[5] Thorell K, Midlöv P, Fastbom J, Halling A. Use of potentially inappropriate medication and polypharmacy in older adults: A repeated cross-sectional study. BMC Geriatr. 2020 Feb; 20: 73. doi: 10.1186/s12877-020-1476-5.

[6] Kucukdagli P, Bahat G, Bay I, Kilic C, Oren MM, Turkmen $\mathrm{BO}$, et al. The relationship between common geriatric syndromes and potentially inappropriate medication use among older adults. Aging Clin Exp Res. 2019 Jun; 32: 681-687. doi: 10.1007/s40520-019-01239-x.

[7] Woodford HJ, Fisher J. New horizons in deprescribing for older people. Age Ageing. 2019 Oct; 48: 768-775. doi: 10.1093/age ing/afz109.

[8] Bratman GN, Hamilton JP, Daily GC. The impacts of nature experience on human cognitive function and mental health. Ann N Y Acad Sci. 2012 Feb; 1249: 118-136. doi: 10.1111/j. 1749-6632.2011.06400.x.

[9] Keniger L, Gaston K, Irvine K, Fuller R. What are the benefits of interacting with nature? Int J Environ Res Public Health. 2013 Mar; 10: 913-935. doi: 10.3390/ijerph10030913.

[10] Hartig T, Mitchell R, Vries S, Frumkin H. Nature and health. Annu Rev Public Health. 2014 Mar; 35: 207-228. doi: 10.1146/annurev-publhealth-032013-182443.

[11] Gascon M, Triguero-Mas M, Martínez D, Dadvand P, Forns J, Plasència $\mathrm{A}$, et al. Mental health benefits of long-term exposure to residential green and blue spaces: A systematic review. Int J Environ Res Public Health. 2015 Apr; 12: 4354-4379. doi: 10.3390/ijerph120404354

[12] McMahan EA, Estes D. The effect of contact with natural environments on positive and negative affect: A meta-analysis. J Posit Psychol. 2015 Jan; 10: 507-519. doi: 10.1080/17439760. 2014.994224.

[13] Sandifer PA, Sutton-Grier AE, Ward BP. Exploring connections among nature, biodiversity, ecosystem services, and human health and well-being: Opportunities to enhance health and biodiversity conservation. Ecosyst Serv. 2015 Apr; 12: 1-15. doi: 10.1016/j.ecoser.2014.12.007.

[14] Depledge MH, Stone RJ, Bird WJ. Can natural and virtual environments be used to promote improved human health and wellbeing? Environ Sci Technol. 2011 Jun; 45: 4660-4665. doi: $10.1021 / \mathrm{es} 103907 \mathrm{~m}$. 
[15] White MP, Yeo NL, Vassiljev P, Lundstedt R, Wallergård M, Albin M, et al. A prescription for "nature" - the potential of using virtual nature in therapeutics. Neuropsychiatr Dis Treat. 2018; 14: 3001-3013.

[16] Slater M. Immersion and the illusion of presence in virtual reality. Br J Psychol. 2018 May; 109: 431-433. doi: 10.1111/ bjop. 12305.

[17] Makransky G, Terkildsen TS, Mayer RE. Adding immersive virtual reality to a science lab simulation causes more presence but less learning. Learn Instr. 2019 Apr; 60: 225-236. doi: 10.1016/j.learninstruc.2017.12.007.

[18] Shu Y, Huang YZ, Chang SH, Chen MY. Do virtual reality head-mounted displays make a difference? A comparison of presence and self-efficacy between head-mounted displays and desktop computer-facilitated virtual environments. Virtual Real. 2018 Nov; 23: 437-446. doi: 10.1007/s10055-018-0376$\mathrm{x}$.

[19] Jeunet C, Albert L, Argelaguet F, Lecuyer A. Do you feel in control?: Towards novel approaches to characterise, manipulate and measure the sense of agency in virtual environments. IEEE Trans Vis Comput Graph. 2018 Apr; 24: 1486-1495. doi: 10.1109/tvcg.2018.2794598.

[20] Slater M. Place illusion and plausibility can lead to realistic behaviour in immersive virtual environments. Philos Trans R Soc Lond B Biol Sci. 2009 Dec; 364: 3549-3557. doi: 10.1098/rstb.2009.0138.

[21] Howard MC. A meta-analysis and systematic literature review of virtual reality rehabilitation programs. Comput Human Behav. 2017 May; 70: 317-327. doi: 10.1016/j.chb.2017.01.013.

[22] Shin H, Kim K. Virtual reality for cognitive rehabilitation after brain injury: A systematic review. J Phys Ther Sci. 2015; 27 : 2999-3002. doi: 10.1589/jpts.27.2999.

[23] Mishkind MC, Norr AM, Katz AC, Reger GM. Review of virtual reality treatment in psychiatry: Evidence versus current diffusion and use. Curr Psychiatry Rep. 2017 Sep; 19: 80. doi: 10.1007/s11920-017-0836-0.

[24] Maples-Keller JL, Bunnell BE, Kim SJ, Rothbaum BO. The use of virtual reality technology in the treatment of anxiety and other psychiatric disorders. Harv Rev Psychiatry. 2017; 25: 103-113. doi: 10.1097/hrp.0000000000000138.

[25] Neri SGR, Cardoso JR, Cruz L, Lima RM, Oliveira RJ, Iversen $\mathrm{MD}$, et al. Do virtual reality games improve mobility skills and balance measurements in community-dwelling older adults? Systematic review and meta-analysis. Clin Rehabil. 2017 Oct; 31: 1292-1304. doi: 10.1177/0269215517694677.

[26] Huygelier H, Schraepen B, Ee R, Abeele VV, Gillebert CR. Acceptance of immersive head-mounted virtual reality in older adults. Sci Rep. 2019; 9: 4519. doi: 10.1038/s41598-01941200-6.

[27] Krippendorff K, Butter R. Semantics: meanings of contexts and artifacts. In: Schifferstein HNJ, Hekkert P, eds. Product Experience. Amsterdam: Elsevier; 2008; pp. 353-376.

[28] Park J, Yim JE. A new approach to improve cognition, muscle strength, and postural balance in community-dwelling elderly with a 3-d virtual reality kayak program. Tohoku J Exp Med. 2015; 238: 1-8. doi: 10.1620/tjem.238.1.
[29] Sakhare AR, Yang V, Stradford J, Tsang I, Ravichandran R, $\mathrm{Pa}$ J. Cycling and spatial navigation in an enriched, immersive $3 \mathrm{~d}$ virtual park environment: A feasibility study in younger and older adults. Front Aging Neurosci. 2019; 11: 218. doi 10.3389/fnagi.2019.00218.

[30] Reynolds L, Rodiek S, Lininger M, McCulley MA. Can a virtual nature experience reduce anxiety and agitation in people with dementia? J Hous Elderly. 2018 Apr; 32: 176-193. doi: 10.1080/02763893.2018.1431583.

[31] Moyle W. Effectiveness of a virtual reality forest on people with dementia: A mixed methods pilot study. Gerontologist 2018 Jun; 58: 478. doi: 10.1093/geront/gnw270.

[32] Bruun-Pedersen JR, Serafin S, Kofoed LB. Restorative virtua environment design for augmenting nursing home rehabilitation. J Virtual Worlds Res. 2016 Dec; 9: 1. doi: 10.4101/jvwr. v9i3.7224.

[33] Orr N, Yeo NL, Dean SG, White MP, Garside R. "It makes you feel that you are there": Exploring the acceptability of virtual reality nature environments for people with memory loss. Geriatrics. 2021 Mar; 6: 27. doi: 10.3390/geriatrics6010027.

[34] Kaplan S. The restorative benefits of nature: Toward an integrative framework. J Environ Psychol. 1995 Sep; 15: 169-182. doi: 10.1016/0272-4944(95)90001-2.

[35] Bengtsson A, Grahn P. Outdoor environments in healthcare settings: A quality evaluation tool for use in designing healthcare gardens. Urban For Urban Green. 2014; 13: 878-891. doi: 10.1016/j.ufug.2014.09.007

[36] Yeo NL, Elliott LR, Bethel A, White MP, Dean SG, Garside R. Indoor nature interventions for health and wellbeing of older adults in residential settings: A systematic review. Gerontologist. 2019 Mar; 60(3): e184-e199. doi: 10.1093/geront/gnz019.

[37] Bowman DA, Kruijff E, LaViola JJ, Poupyrev I. 3D User Interfaces: Theory and Practice. 2nd ed. Boston: Addison Wesley; 2017.

[38] Keshavarz B, Hecht H, Lawson BD. Visually Induced Motion Sickness. In: Hale KS, Stanney KM, eds. Handbook of Virtual Environments: Design, Implementation, and Applications. 2nd ed. Boca Raton (FL): CRC Press; 2014; pp. 647-698.

[39] Denzin NK. The research act: a theoretical introduction to sociological methods. 2nd ed. New York: McGraw-Hill; 1978.

[40] Graneheim UH, Lundman B. Qualitative content analysis in nursing research: Concepts, procedures and measures to achieve trustworthiness. Nurse Educ Today. 2004 Feb; 24: 105-112. doi: 10.1016/j.nedt.2003.10.001.

[41] Narushima M, Liu J, Diestelkamp N. The association between lifelong learning and psychological well-being among older adults: Implications for interdisciplinary health promotion in an aging society. Act Adapt Aging. 2013 Jul; 37: 239-250. doi: 10.1080/01924788.2013.816834.

[42] Narushima M, Liu J, Diestelkamp N. Lifelong learning in active ageing discourse: its conserving effect on wellbeing, health and vulnerability. Ageing Soc. 2016 Nov; 38: 651-675. doi: 10.1017/s0144686x16001136.

[43] Phelan A, McCormack B. Exploring nursing expertise in residential care for older people: A mixed method study. J Adv Nurs. 2016 Oct; 72: 2524. doi: 10.1111/jan.13001. 